\title{
Le ipernatriemie: cause e gestione
}

\author{
Elisa Selmin ${ }^{1} \cdot$ Filippo Ceccato $^{1} \cdot$ Carla Scaroni $^{1}$
}

Accettato: 27 maggio 2020 / Pubblicato online: 2 giugno 2021

(C) The Author(s) 2021

\section{Definizione e epidemiologia}

Per ipernatriemia si intende una concentrazione di sodio plasmatico $>145 \mathrm{mmol} / \mathrm{L}$. Di frequente riscontro nelle corsie ospedaliere, nell' ambito della terapia intensiva incrementa il tasso di mortalità fino al 40\% e aumenta i tempi di degenza del 28\%, in particolare se severa (>156 mmol/L) [1]. L'approccio al paziente con ipernatriemia si basa su: 1) diagnosi iniziale; e 2) scelta e modalità del trattamento.

\section{Nosografia}

Causa frequente di ipernatriemia è la perdita di acqua in eccesso rispetto agli elettroliti. Altre cause sono la fuoriuscita di acqua pura o di fluidi ipotonici, più raramente l'assunzione di sodio in eccesso (soluzione ipertonica). Perché l'ipernatriemia risulti cronica è necessario che coesista un alterato meccanismo della sete $\mathrm{o}$ una preclusione alla disponibilità di acqua.

La perdita netta di acqua può verificarsi per cause renali e cioè per Diabete insipido (DI) centrale o nefrogenico oppure per perdita renale di Na: diuretici dell'ansa, diuresi osmotica (iperglicemia, mannitolo, urea elevata), postostruttiva, poliuria post necrosi tubulare acuta, danno renale intrinseco.

Le ipernatriemie da cause non-renali possono essere determinate da: 1) ipodipsia; 2) aumentata perspiratio insensibilis non compensata; o 3) perdita gastrointestinale: vomito, diarrea, uso di terapia catartica osmotica, sondino naso-gastrico.

$\triangle$ F. Ceccato

ceccato.filippo@gmail.com

1 U.O. di Endocrinologia, Dipartimento di Medicina DIMED, Azienda Ospedale-Università di Padova, Padova, Italia
Tabella 1 Concentrazione di sodio nelle infusioni più frequentemente utilizzate [2]

\begin{tabular}{lc}
\hline Infusione & $\mathrm{Na}^{+}$somministrato (mmol/L) \\
\hline Soluzione glucosata 5\% (destrosio) & 0 \\
Sodio cloruro 0,2\% in glucosata 5\% & 34 \\
Soluzione fisiologica 0,45\% & 77 \\
Ringer lattato & 130 \\
Soluzione fisiologica 0,9\% & 154 \\
\hline
\end{tabular}

Più raramente, l'ipernatriemia può essere conseguente a un eccesso di sodio, dovuto a: 1) ingestione di alimenti/liquidi ricchi di sodio; ovvero a 2) somministrazione iatrogena (es. soluzione ipertonica, sodio bicarbonato).

In condizioni particolari l'ipernatriemia può essere secondaria ad attivazione del recettore dei mineralcorticoidi, dove si accompagna a ipopotassiemia, come ad esempio in caso di:

- iperaldosteronismo primario;

- sindrome di Cushing;

- ipertensioni monogeniche a bassa renina.

\section{Diagnosi iniziale}

La sintomatologia si distingue per alterazione dello stato mentale fino al coma, astenia, debolezza muscolare, convulsioni. In particolare, in caso di ipernatriemia acuta (esordio $<48 \mathrm{~h}$ ), l'improvvisa riduzione di volume delle cellule cerebrali può provocare emorragie parenchimali o subaracnoidee e/o ematomi subdurali, soprattutto nel paziente pediatrico. Inoltre, il danno alle membrane muscolari può provocare rabdomiolisi ipernatriemica [1]. L'ipernatriemia, infatti, aumenta l'osmolalità del fluido extracellulare ( $>295 \mathrm{mOsml} / \mathrm{Kg}$ ), con creazione di un gradiente osmo- 
Tabella 2 Calcolo del deficit idrico (formula 1), della clearance dell'acqua libera (formula 2) e dell'infusione di liquidi nel trattamento dell'ipernatriemia plasmatica (formule 3 e 4) [2, 3].
$V$, volume urinario; $U_{\mathrm{Na}}$, concentrazione di Na nelle urine; $U_{\mathrm{K}}$, concentrazione $\mathrm{K}$ nelle urine

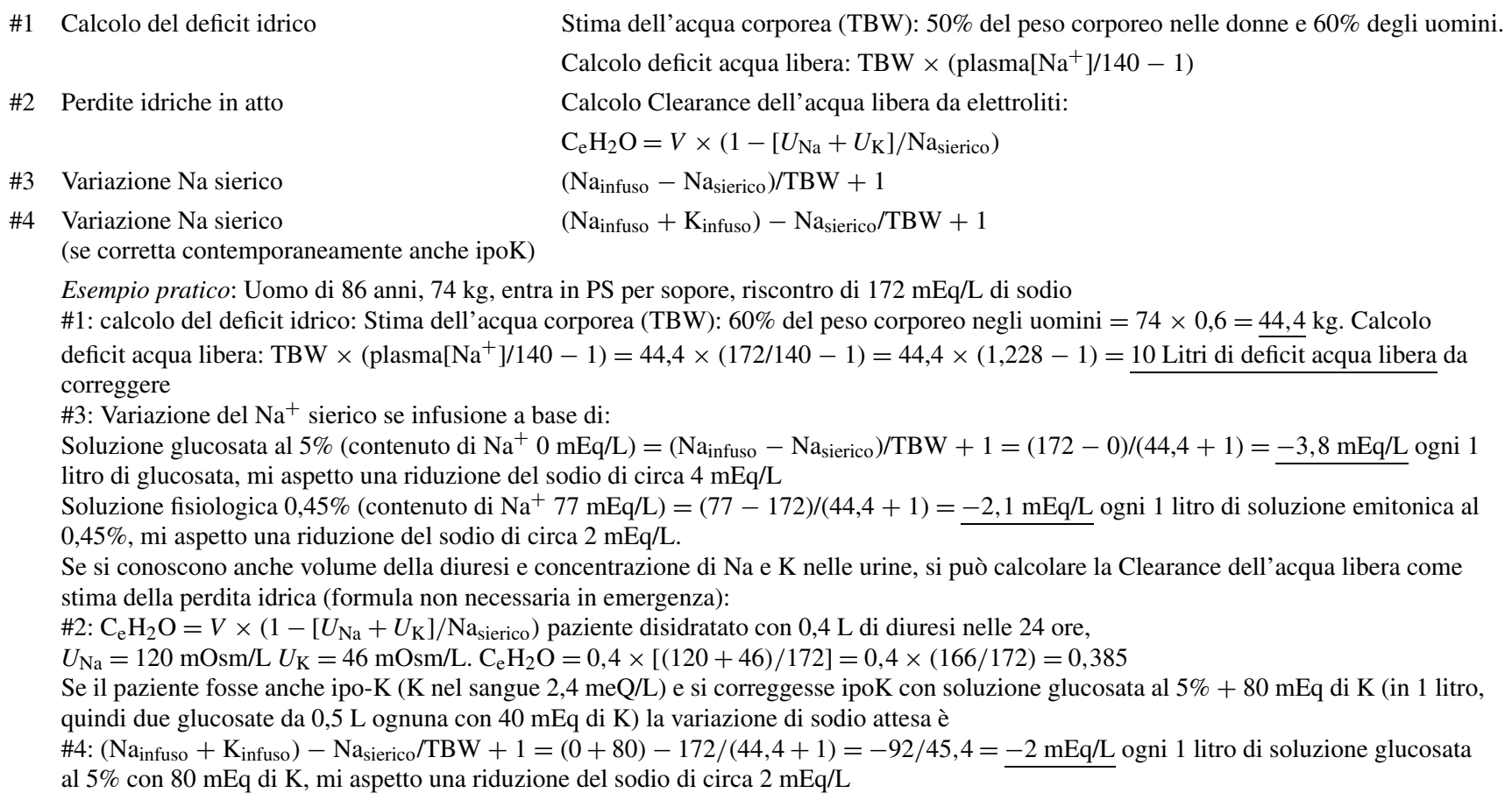

tico fra fluido extra- e intra-cellulare e conseguente flusso d'acqua all'esterno delle cellule, con contrazione cellulare. L'ipernatriemia cronica (esordio $>48$ ore) è spesso asintomatica o si manifesta con sintomatologia lieve, in quanto sono presenti meccanismi di adattamento a livello delle cellule cerebrali che ne limitano la disidratazione.

Per il corretto inquadramento diagnostico sono importanti l'anamnesi (pregressi traumi cerebrali, terapia antidepressiva con litio) e l'esame obiettivo del paziente (valutazione della volemia del paziente: ipovolemia per perdita di acqua e sali; normovolemia per perdita di sola acqua; ipervolemia per eccessiva introduzione di sodio). È inoltre fondamentale, oltre alla misurazione del sodio plasmatico, il calcolo della osmolalità plasmatica e urinaria.

\section{Scelta e modalità del trattamento}

Per trattare l'ipernatriemia, fondamentale è identificare e rimuovere una causa correggibile, se presente, impostando una terapia mirata. È importante, inoltre, verificare se l'ipernatriemia sia acuta o cronica. La forma acuta può richiedere una correzione dei valori di natriemia fino a $1 \mathrm{mmol} / \mathrm{l} / \mathrm{h}$. Nella forma cronica la correzione deve essere lenta, non superando l'incremento di $10 \mathrm{mmol} / \mathrm{l}$ nelle 24 ore soprattutto nelle forme gravi; in caso contrario, può insorgere edema cerebrale con conseguenti convulsioni, alterazioni dello stato di coscienza fino al coma. Il rischio di edema cerebrale aumenta proporzionalmente al volume delle infusioni somministrate [2]. La terapia consiste nell'integrazione con acqua libera per os, o infusione di soluzioni ipotoniche (es. sodio cloruro $0,45 \%$ o glucosata $5 \%$, monitorando la glicemia) (Tabella 1).

Nelle ipernatriemie da disidratazione si deve inizialmente calcolare il deficit idrico (Tabella 2, formula 1). La stima dell' acqua totale corporea (in litri) è calcolata come una frazione del peso corporeo (0,6 nei bambini, 0,6 e 0,5 rispettivamente in uomini e donne adulti), distribuito per il $40 \%$ in regione extracellulare e $60 \%$ intracellulare.

In seguito deve essere calcolata la velocità di somministrazione dell'infusione. La formula no. 3 stima l'effetto della somministrazione di un litro di qualsiasi infusione sul sodio sierico. La formula no. 4 permette di calcolare l'effetto della somministrazione di un litro di infusione contenente sia sodio che potassio sulla concentrazione di sodio sierico del paziente.

Per calcolare la velocità di somministrazione dell'infusione scelta, si divide la stima della variazione della concentrazione di sodio sierico desiderata per il tempo necessario per la correzione della stessa, in sicurezza (es. per ottenere una riduzione di $8 \mathrm{mEq}$ di sodio nelle 24 ore con la infusione scelta, devo considerare di infondere a $1000 \mathrm{~mL} / 24 \mathrm{~h}=$ $40 \mathrm{cc} / \mathrm{h}$, oppure per ottenere la stessa riduzione in $\mathrm{mEq}$ di sodio, ma in 36 ore, devo calcolare $1000 \mathrm{~mL} / 36 \mathrm{~h}=25 \mathrm{cc} / \mathrm{h}$ ) [2]. 
Funding Note Open access funding provided by Università degli Studi di Padova within the CRUI-CARE Agreement.

Conflitto di interesse Gli autori Elisa Selmin, Filippo Ceccato e Carla Scaroni dichiarano di non avere conflitti di interesse.

Consenso informato Lo studio presentato in questo articolo non ha richiesto sperimentazione umana.

Studi sugli animali Gli autori dichiarano di non aver eseguito studi sugli animali.

Nota della casa editrice Springer Nature rimane neutrale in riguardo alle rivendicazioni giurisdizionali nelle mappe pubblicate e nelle affiliazioni istituzionali.

Open Access This article is licensed under a Creative Commons Attribution 4.0 International License, which permits use, sharing, adaptation, distribution and reproduction in any medium or format, as long as you give appropriate credit to the original author(s) and the source, provide a link to the Creative Commons licence, and indicate if changes were made. The images or other third party material in this article are included in the article's Creative Commons licence, unless indicated otherwise in a credit line to the material. If material is not included in the article's Creative Commons licence and your intended use is not permitted by statutory regulation or exceeds the permitted use, you will need to obtain permission directly from the copyright holder. To view a copy of this licence, visit http://creativecommons.org/licenses/by/4.0/.

\section{Bibliografia}

1. Muhsin SA, Mount DB (2016) Diagnosis and treatment of hypernatremia. Best Pract Res, Clin Endocrinol Metab 30(2):189-203

2. Adrogué HJ, Madias NE (2000) Hypernatremia. N Engl J Med 342(20):1493-1499

3. Longo D, Fauci A, Kasper D et al (2012) Ipernatriemia. In: Harrison (ed) Principi di Medicina Interna, 18a edn. Casa Editrice Ambrosiana, Milano, pp 351-353 